\title{
La motte castrale de Guéramé à Courgains (Sarthe), aux confins du Maine et du Perche
}

The Guéramé castle motte of Gourgains (Sarthe) on the borders of Maine and Perche

Alain Valais, Ludovic Schmitt et Emmanuelle Coffineau

Traducteur : Marc Duma et Jean-François Nauleau

\section{(2) OpenEdition}

Journals

Édition électronique

URL : https://journals.openedition.org/rao/1368

DOI : 10.4000/rao.1368

ISSN : 1775-3732

Éditeur

Presses universitaires de Rennes

Édition imprimée

Date de publication : 31 décembre 2010

Pagination : 149-170

ISBN : 978-2-7535-1383-9

ISSN : 0767-709X

Référence électronique

Alain Valais, Ludovic Schmitt et Emmanuelle Coffineau, "La motte castrale de Guéramé à Courgains (Sarthe), aux confins du Maine et du Perche», Revue archéologique de l'Ouest [En ligne], 27 | 2010, mis en ligne le 25 février 2012, consulté le 22 août 2022. URL : http://journals.openedition.org/rao/1368 ; DOI : https://doi.org/10.4000/rao.1368

Ce document a été généré automatiquement le 22 août 2022.

Tous droits réservés 


\title{
La motte castrale de Guéramé à Courgains (Sarthe), aux confins du Maine et du Perche
}

\author{
The Guéramé castle motte of Gourgains (Sarthe) on the borders of Maine and \\ Perche
}

Alain Valais, Ludovic Schmitt et Emmanuelle Coffineau

Traduction : Marc Duma et Jean-François Nauleau

Une opération de diagnostic réalisée en 2006 sur la commune de Courgains dans le nord de la Sarthe a permis de confirmer la présence d'une motte et de sa basse-cour, site découvert en prospection aérienne en 1999 par G. Leroux (INRAP). Le gisement, baptisé « Guéramé » par son inventeur, est d'abord constitué d'un fossé de 7 à $8 \mathrm{~m}$ de largeur et de presque $2,50 \mathrm{~m}$ de profondeur. Il entoure une plate-forme circulaire de $20 \mathrm{~m}$ de diamètre. Aujourd'hui, le relief de la motte ainsi enserrée est presque totalement altéré. Le cœur du site est associé à d'autres fossés qui limitent probablement deux états de basse-cour. En plus de fossés de partition, l'emprise étudiée a livré des trous de poteau plutôt concentrés vers la motte. Parmi les quelques fosses relevées, une structure rectangulaire retient l'attention avec un trou de poteau à chacun de ses angles.

Le mobilier issu des quelques structures testées se compose de quelques fragments de métal dont de possibles éléments de broigne ou d'armure. Le corpus céramique bien que modeste atteste néanmoins d'une occupation centrée sur les XII ${ }^{e}$ et XIII ${ }^{e}$ siècles.

Plus largement, le site de Guéramé s'inscrit au centre d'un système parcellaire concentrique, en partie conservé sur les relevés cadastraux du XIX ${ }^{e}$ siècle. Cet ensemble s'appuie à l'est sur une voie ancienne près de laquelle est établie une seconde motte, celle de Concé distante de seulement $300 \mathrm{~m}$. Ce chemin relie vers le nord Saosnes puis plus loin Saint-Rémi-en-Val (ancien Saint-Rémi-du-Plan), et vers le sud la butte du Theil et le Peray, quatre sites où subsistent également des mottes. Un texte de 1098 indique que Robert II, seigneur de Bellême et vassal du duc de Normandie, fortifie le Saosnois contre les velléités du comte du Maine. Il entame, sur ses terres mais aussi sur celles de 
ses vassaux, la réparation et l'édification de complexes castraux dont il dresse la liste. Une étude documentaire a permis également de retrouver un lignage de Guéramé qui occupait le site diagnostiqué et dont les membres sont attestés du début du XII ${ }^{\mathrm{e}}$ siècle au début du siècle suivant.

2 Trial excavations, undertakent in 2006 in the commune of Courgains (north of Sarthe), confirmed the presence of a motte and its bailey on a site discovered from the air in 1999 by G. Leroux (INRAP) and recorded by him under the name of "Guéramé". It mainly consists of a ditch 7 to $8 \mathrm{~m}$ wide and nearly $2,5 \mathrm{~m}$ deep, enclosing a circular platform $20 \mathrm{~m}$ in diametre, the whole being today almost completely levelled. Other associated ditches delimit a probably two phased bailey. In addition to partition ditches, the studied area yelded post-holes, mainly concentrated near the motte. Among various pits recognized, a rectangular one, with post-holes at each corner, is noteworthy.

Artefacts yelded by the few structures examined includes some metal fragments, among which two possible elements of scale armour. The ceramic assemblage, although small, attests to an occupation centered on 12 th and $13^{\text {th }}$ centuries.

More widely, the site of Guéramé appears central to a concentric field system which was still partly preserved on 19th century cadastral plans. At the east, this complex is defined by an ancient track near which stands a second motte, Concé, only $300 \mathrm{~m}$ away. This track joins four other motte sites (Saosnes and St. Rémy-en-Val northwards; Le Theil and Le Perray southwards). According to a text of 1098, Robert II, lord of Bellême and vassal of the Duke of Normandy, fortified Saosnois area against the Earl of Maine's ambitions. On his own land as well as on that of his vassals, he undertook repairs and buildings of castral complexes, each summarized in the text. An archival study also revealed a Guéramé lineage occupying this site, whose membres are attested from the early 12 th century to the beginning of the next.

\section{Présentation de l'opération}

\section{Circonstances de la découverte et présentation du site}

Le site de Guéramé a été découvert sur la commune de Courgains en 1999 par G. Leroux (inrap) au cours d'une de ses missions aériennes (fig. 1 et 2), mais cette motte castrale est enregistrée à la carte archéologique sous le nom de « la Colinière » $\left(n^{\circ} 72.104 .0014\right.$; fig. 3, n 14). En février 2006, les parcelles concernées par les vestiges ont été soumises à un diagnostic archéologique avant aménagement d'une zone artisanale. L'opération a conduit à la modification du projet en excluant le périmètre occupé par les vestiges enfouis. Les données dont il sera question tout au long de cet article sont donc issues de cette seule phase de diagnostic.

\section{Localisation}

Le site archéologique est implanté au nord du bourg de Courgains, à $500 \mathrm{~m}$ de l'église paroissiale du village. La zone appartient au versant nord de la vallée du ruisseau de la Malherbé. Les terrains sont peu accidentés mais une pente assez régulière existe entre les extrémités sud-ouest et nord-est du projet; cette dernière culmine à près de $119 \mathrm{~m}$ d'altitude et les parties les plus basses n'atteignent qu'un peu moins de $109 \mathrm{~m}$. Un 
talweg existe le long de sa limite nord-ouest. Cette vallée semble avoir été en partie colmatée par des colluvions ; c'est en tout cas ce que montrent deux sondages ouverts dans ce secteur (sondage 68 et extrémité ouest du sondage $11:$ fig. 4). L'existence de cette phase de remblaiement est antérieure à un fossé (F 9 , sondage 11), encore mentionné sur les planches du cadastre de 1837 (feuille A3).

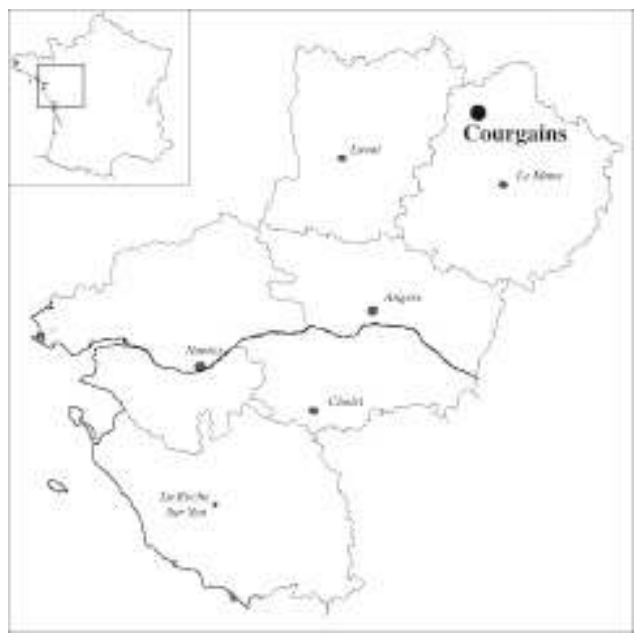

Figure 1 : Localisation générale de Courgains (sauf précision, toutes les illustrations sont de J.-F. Nauleau).

Figure 1: General location map (unless precised, all illustrations are by J.-F. Nauleau).

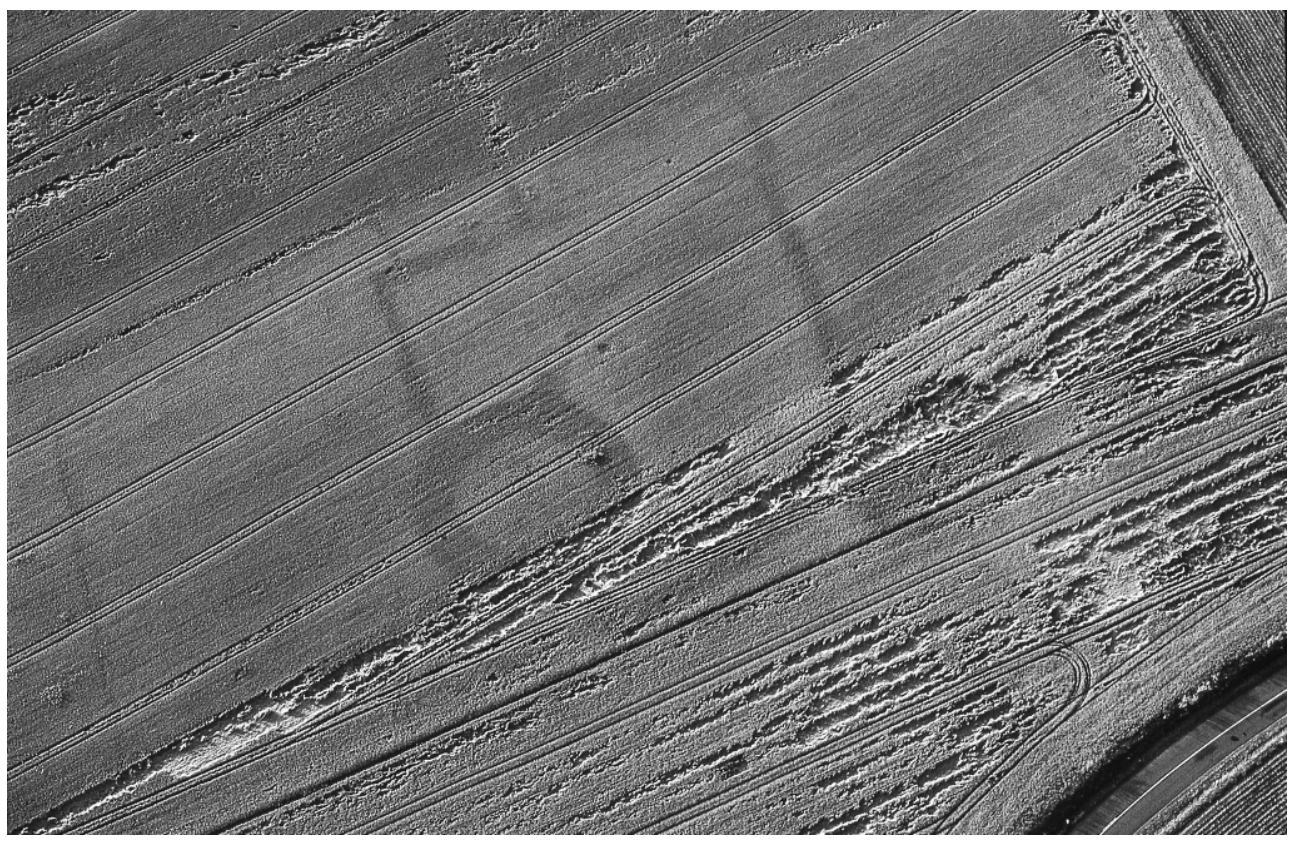

Figure 2: Vue aérienne du site de Guéramé en 1999 lors de sa découverte (photo G. Leroux, INRAP). Figure 2: Air view of the site in 1999.

5 Le parcellaire autour du village de Courgains a été totalement démantelé à la suite d'une très lourde campagne de remembrement du début des années 1980. Des riverains nous ont signalé que ces travaux avaient été réalisés au bulldozer et par temps de pluie. Depuis, les parcelles du projet ont été totalement cultivées et, aujourd'hui, leur surface ne présente plus que de légères ondulations dont la nature reste difficile à identifier. 


\section{Contexte géologique}

6 Le sous-sol se compose d'« oolithes ferrugineuses de Chemilly»(J3b1 - callovien inférieur $)^{1}$ qui se caractérisent par des calcaires argileux bruns clairs. Il semble également que nous ayons rencontré des « horizons de Saint-Calez-en-Saosnois » (J3b2 - Callovien moyen), le plus souvent argileux brun clair ou beige rosé. Des fossiles ont été observés dans tous ces horizons. Dans quelques sondages, des marnes grises ont été atteintes, parfois sous au moins deux mètres de limons (sondages 1 et 2 par exemple).

\section{Contexte archéologique}

$7 \quad$ L'indice de site repéré par G. Leroux (fig. $3, \mathrm{n}^{\circ} 14$ ) se compose d'un ensemble de fossés dont le plus large (plus de $5 \mathrm{~m}$ ) enserre une surface parfaitement circulaire d'environ $20 \mathrm{~m}$ de diamètre (fig. 2). Ce réseau est connecté à des limites de gabarit plus modeste qui circonscrivent un enclos rectangulaire à partition interne. Le complexe a été interprété par son inventeur comme un exemple de motte castrale que complètent plusieurs états de basse-cour. En dehors de quelques anomalies dans le tracé de haies représentées sur le cadastre napoléonien, rien sur le terrain ne subsiste de ce site.

Les autres découvertes recensées sur la commune sont tout aussi intéressantes. Une autre motte castrale existe à Concé (fig. $3, \mathrm{n}^{\circ} 2$ ), à seulement $300 \mathrm{~m}$ au sud-est de l'indice de Guéramé ; elle conserve une douve qui entoure une plate forme de $45 \mathrm{~m}$ de diamètre. Une ferme appelée Le ferrier $\left(\mathrm{n}^{\circ} 4\right)$ est également mentionnée à $200 \mathrm{~m}$ au sud du site de Concé ; ce toponyme témoigne d'une activité métallurgique difficile à dater. Plus loin vers le sud-est (à $1 \mathrm{~km}$ environ), la butte du Theil, appelée également Mont de la Garde, est couronnée par un autre exemplaire de motte castrale (fig. $3, n^{\circ} 1$ ). Ce retranchement domine la région de Courgains depuis les rebords d'une cuesta. Il est établi à l'angle d'un éperon barré défendu par un fossé associé à un rempart double. La datation de cet ensemble de 5,8 hectares reste imprécise (Bouvet, 2001, p. 215).

Parmi les autres sites de la commune, on dénombre des enclos rectilinéaires (fig. $3, \mathrm{n}^{\circ} 5$ et 13) ou curvilinéaires protohistoriques $\left(\mathrm{n}^{\circ} 9,10\right.$ et 16$)$, ainsi que quelques implantations antiques : un fanum aux Noes $\left(n^{\circ} 7\right)$ et un bâtiment de la même période à la Bâtisse $\left(n^{\circ} 8\right)$.

\section{L'opération de sondages}

10 En plus de deux sondages perpendiculaires réalisés sur le site de Guéramé, toutes les parcelles concernées par le projet d'aménagement ont été explorées par des tranchées de $20 \mathrm{~m}$ de longueur ouvertes en quinconce sur des bandes espacées de $20 \mathrm{~m}$ également (fig. 4). Ainsi, ce sont environ $8 \%$ de la surface qui ont été explorés. Sur l'emprise de l'indice, nous nous sommes efforcés d'ouvrir les terrains le moins possible car, dans l'optique d'une fouille ultérieure, il importait de limiter l'impact de nos sondages forcément destructeurs. 


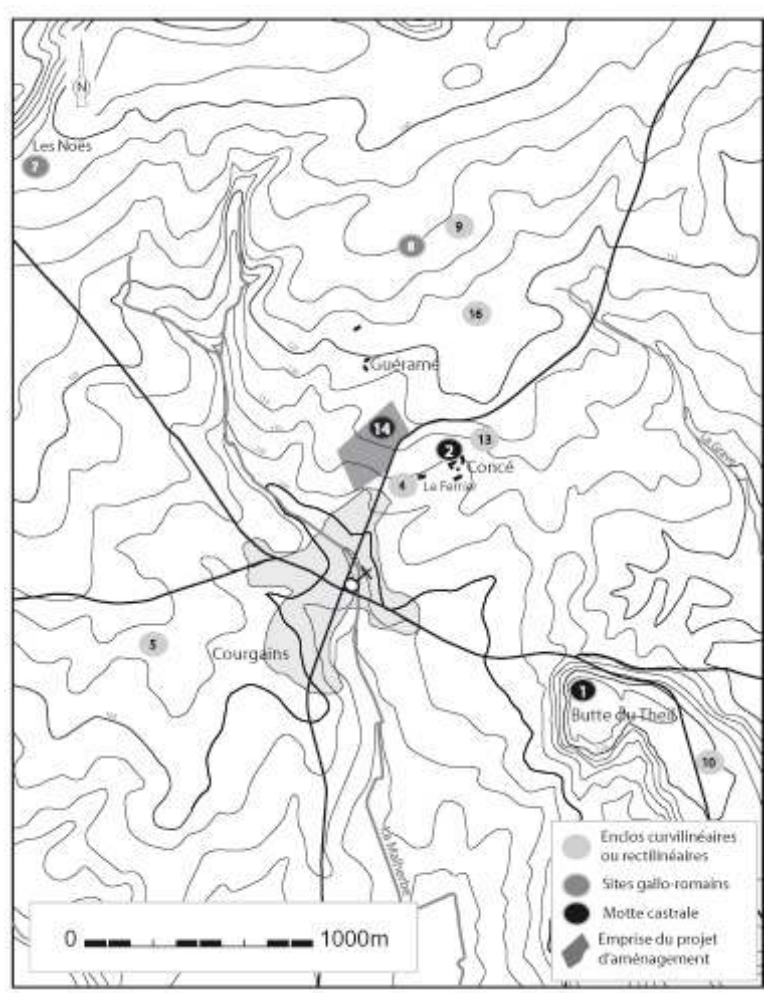

Figure 3 : Localisation du projet d'aménagement et des sites voisins évoqués (à partir du fond topographique IGN à 1/25 000)

Figure 3: Location of land improvement project and of neighbouring sites mentionned.

\section{Un site fossoyé médiéval}

\section{Une occupation médiévale évidente}

En dehors de quelques fossés protohistoriques et antiques qui paraissent bien isolés, toutes les informations recueillies au cours de l'opération concernent le Moyen Âge. Il s'agit d'abord d'un fossé de gabarit imposant et d'une mare, deux éléments facilement identifiables sur la photographie aérienne. Ces deux creusements spectaculaires, d'abord identifiés dans le sondage 3 (fig. 4), nous ont permis de nous situer sur le plan redressé du gisement reconnu du ciel.

\section{Le fossé principal}

Le fossé le plus imposant a été repéré dans les sondages 3, 12 et 13 (à deux reprises) (fig. 5). Il mesure environ $7 \mathrm{~m}$ de largeur dans les sondages 3 et $12^{2}$. Cet ensemble décrit un cercle d'environ $34 \mathrm{~m}$ de diamètre externe et enserre une surface non excavée de $20 \mathrm{~m}$ de diamètre. Une seule coupe a été réalisée dans ce fossé, au niveau du sondage $\mathrm{n}^{\circ} 3$. Son profil est évasé avec un fond plat de près de 2,50 $\mathrm{m}$ de largeur. La profondeur du fossé atteint un peu plus de 1,60 m par rapport au niveau de décapage, mais il faut lui ajouter $0,90 \mathrm{~m}$ jusqu'à la surface actuelle de la parcelle. La douve devait ainsi mesurer près de $2,50 \mathrm{~m}$ de profondeur. 


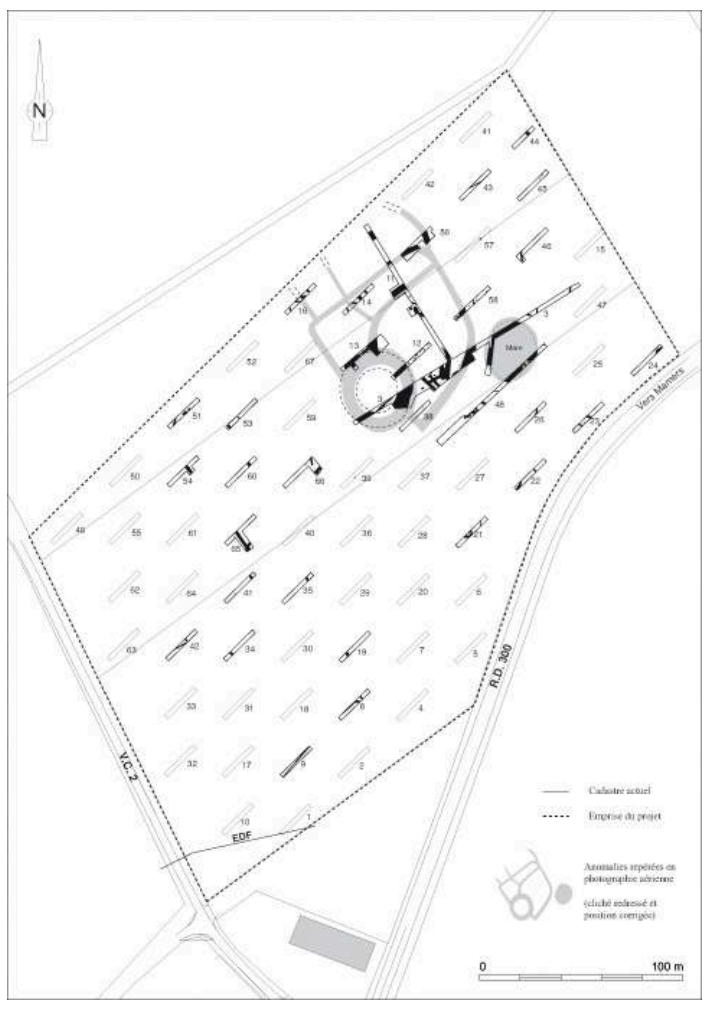

Figure 4 : Plan général du diagnostic avec localisation des structures découvertes.

Figure 4: General plan of excavations and location of uncovered features.

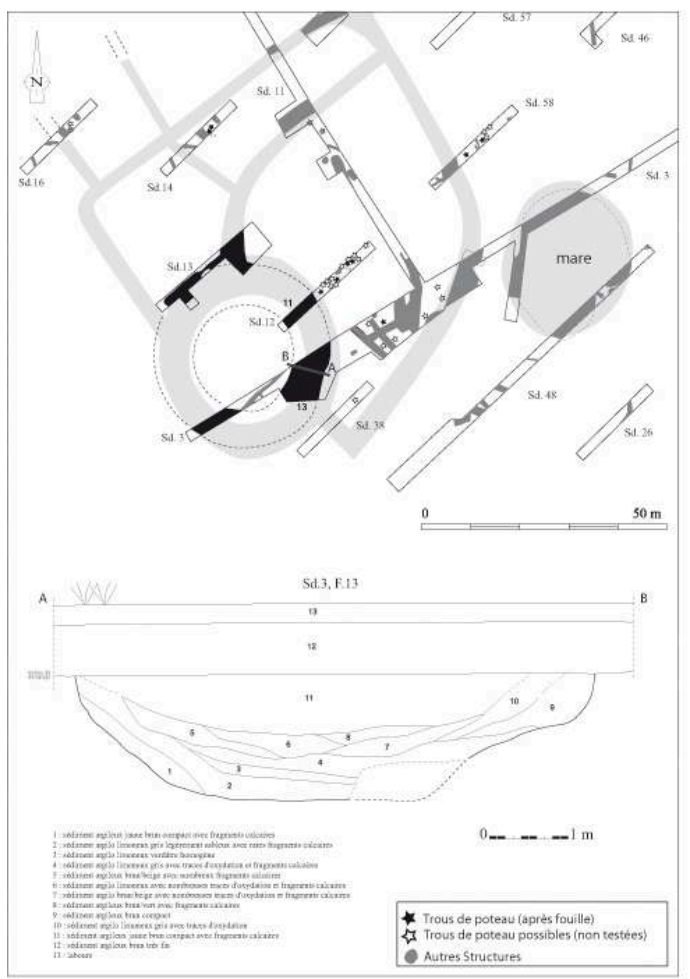

Figure 5 : Fossés et autres structures en creux de la motte; Coupe $A B$ dans le fossé de la motte. Figure 5: Ditches and other subsoil features of motte; $A B$ section of motte ditch.

13 Ses phases initiales de remplissage (us 1 à 10) couvrent la durée d'occupation du site. Les apports, constitués par les us 1 et 2, proviennent de l'extérieur du fossé. Ces 
horizons sont probablement issus de l'altération des parois du creusement. Ce phénomène n'apparaît pas sur le versant intérieur de l'enclos, espace probablement mieux stabilisé. L'origine des phases de remplissage postérieures semble ensuite s'équilibrer à partir de l'us 4 . Les horizons, depuis l'us 1 jusqu'à l'us 10 , sont très peu organiques. Ils sont constitués de sédiments argileux plus ou moins riches en inclusions calcaires. Le mobilier archéologique en est totalement absent.

Les parties supérieures du remplissage (US 11) sont plus homogènes (des limons argileux compacts). Elles correspondent à une importante phase de remblaiement du fossé. On ne sait à quelle période faire remonter ce nivellement qui résulte probablement de l'arasement de la motte. Celle-ci n'apparaît plus ni sur le cadastre de 1837 (ADS, PC/105/013) ni même sur un plan terrier de la fin du XVIII ${ }^{e}$ siècle (ADS, E 316-13).

\section{D'autres fossés de fort gabarit}

Des fossés importants ont été relevés dans les sondages 3 (F 5), 13 (F 2), 11 (F 6), 56 (F 1 et $\mathrm{F} 2$ ) et 58 ( $\mathrm{F} 9$ ) (fig. 6). Les largeurs varient de $2,20 \mathrm{~m}$ au moins pour $\mathrm{F} 56.1$ à environ $4,50 \mathrm{~m}$ pour $\mathrm{F}$ 13.2. Des profils ont été observés dans tous ces fossés, excepté $\mathrm{F} 13.2$ et F 58.9 qui n'ont pas été sondés; ils sont à fond plat et dotés de bords assez évasés. Les profondeurs varient de $1 \mathrm{~m}$ (F 56.1 et F 56.2) à $1,55 \mathrm{~m}$ pour F 3.5. Ce dernier est le plus profond; il présente, contrairement aux deux autres, un profil dissymétrique lié sans doute à un recreusement identifiable en stratigraphie.

Les phases de remplissage sont constituées majoritairement de sédiments argileux compacts et homogènes avec des quantités variables de petits blocs de calcaire. Ces fossés ont livré peu de mobilier; citons un fragment de tuile canal médiévale dans le fossé 3.5 et un petit lot céramique des $\mathrm{XII}^{\mathrm{e}}$-XIII ${ }^{\mathrm{e}}$ siècles dans le fossé 11.6.

On peut identifier la plupart de ces importants fossés sur la photo aérienne de référence (fig. 2). Ces limites appartiennent à un enclos qui vient de greffer sur l'enclos circulaire décrit plus haut. Un seul des deux points de contact a été retrouvé (dans le sondage 13). Le fossé 13.2 semble se poursuivre dans le sondage 11 par F 6 , tout comme, à proximité, le fossé 56.1 dans le sondage 11 avec $\mathrm{F} 5$. Il est probable également que le fossé 56.2 soit la prolongation de F 58.9 et, plus loin, du tronçon F 3.5. Le retour de cette limite, évidente sur le cliché aérien, se situe entre les sondages 3 et 48 . Il est probable, mais nous n'avons pas pu le vérifier, que le fossé ait été scellé sous une vaste anomalie relevée dans la moitié nord est du sondage 38 , juste avant de rentrer en contact avec l'enclos principal.

\section{Des fossés plus modestes}

18 Les autres fossés du secteur présentent de moindres gabarits (fig. 6). Plusieurs ont été relevés dans les sondages 3 et 11. Il s'agit de fossés $(11.1,11.6,3.7)$ qui mesurent entre 1,60 et 2,50 $\mathrm{m}$ de largeur. Le seul qui ait été testé ne dépasse pas $0,20 \mathrm{~m}$ de profondeur conservée (F 11.1). Les fossés 11.1 et 3.6, avec des orientations voisines, s'interrompent vers le nord. Au sud, F 11.1 pourrait se poursuivre dans le sondage 3 avec le fossé 7. De son côté, F 3.6 pourrait se prolonger au sud du sondage 3 avec F 3.19. 


\section{Fossés isolés ou non datés}

Deux fossés médiévaux isolés doivent être signalés. Le premier a été relevé dans le sondage 51 (fig. 4); il mesure $1 \mathrm{~m}$ de largeur. Le second appartient au sondage voisin (Sd. 54); il mesure 1,50 m de largeur et 0,60 $\mathrm{m}$ de profondeur et son profil est en V. Le mobilier issu du sondage 54 appartient au xiII ${ }^{\mathrm{e}}$ siècle et celui recueilli dans le fossé du sondage 51 est attribuable aux $\mathrm{XII}^{\mathrm{e}}$-XIII ${ }^{\mathrm{e}}$ siècles. Malgré leur proximité, leurs orientations divergentes ne permettent pas de relier ces deux fossés. Ils pourraient toutefois marquer la limite du site vers le sud. On peut sans doute également leur associer le fossé 2 dans le sondage 65. Malgré un tesson roulé d'allure plutôt protohistorique trouvé dans le remplissage de $\mathrm{F} 1$, il est possible, du fait de leur orientation commune, de rapprocher de ce fossé du sondage 54 le fossé 2 du sondage 65, qui remonte assurément à la période médiévale...

Les autres fossés du secteur sont nombreux. Malheureusement, beaucoup n'ont livré aucun mobilier. On peut citer pour mémoire ceux du sondage 16 (trois exemplaires non datés, le quatrième étant mentionné sur les planches du cadastre) (fig. 6), du sondage 14 (trois exemplaires non datés), du sondage 58 (trois fossés en dehors de F 9) et de l'extrémité nord-est du sondage 3 (trois exemplaires également), sans compter ceux de la tranchée 48. Notons en outre le fossé découvert dans chacun des sondages $43,57,46$ et 26 .

\section{Les autres structures}

\section{Une mare}

21 Une mare a été relevée dans le sondage 3 (fig. 5); celle-ci apparaît déjà sur la photo aérienne (fig. 2). Il s'agit d'un creusement qui mesure jusqu'à $2 \mathrm{~m}$ de profondeur sous la surface actuelle de la parcelle et dont le diamètre dépasse $27 \mathrm{~m}$. Son remplissage est constitué à la base d'un niveau de limon argileux gris, riche en graviers. Cet horizon initial d'un mètre d'épaisseur est imprégné par les eaux d'une ou plusieurs sources. Cette phase humide est scellée sous un horizon de limon argileux oxydé où de la faune et un peu de mobilier céramique ont été extraits. Les deux niveaux supérieurs s'apparentent à des couches de colmatage remontant probablement au rebouchage de cette mare dont l'origine médiévale ne fait pas de doute, le mobilier recueilli dans la phase de remplissage inférieur appartenant aux XII ${ }^{\mathrm{e}}$-XIII ${ }^{\mathrm{e}}$ siècles.

Revue archéologique de l'Ouest, 27 | 2010 


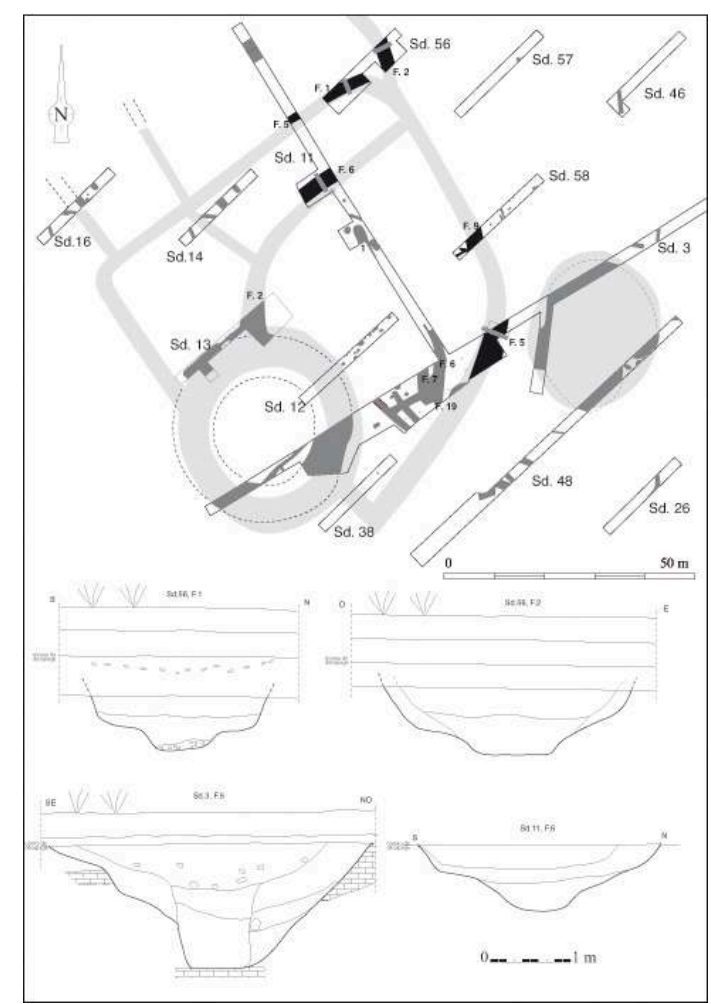

Figure 6: Coupes des fossés de la basse-cour et leur localisation.

Figure 6 : Sections of bailey ditches and their location.

Ce point d'eau, alimenté par des sources, permettait très probablement de remplir les douves du site médiéval implanté juste en aval, l'enceinte de la motte étant sans doute liée à la mare par le biais du fossé 5 du sondage 3 (fig. 6), bien que la connexion n'ait pas été localisée.

\section{Des trous de poteaux}

Les trous de poteaux retrouvés se concentrent dans les sondages implantés immédiatement au nord et au nord-est de l'enclos circulaire (fig. 5). Le sondage 12 en a livré une douzaine à lui seul; ils mesurent entre $0,20 \mathrm{~m}$ et un peu moins de $1 \mathrm{~m}$ de diamètre. Les quatre qui y ont été sondés montrent des états de conservation médiocres, entre quelques centimètres et une quinzaine de centimètres de profondeur conservée. D'autres trous ont été relevés, également en assez grand nombre, dans les sondages 3 et 58. D'autres, plus rares, apparaissent en outre dans les sondages 14, 16, 45 et 23 .

Outre que ces structures appartiennent à un secteur occupé durant la période médiévale, la découverte d'un peu de mobilier dans quelques-unes d'entre-elles vient confirmer leur appartenance à cette même période. Le creusement F. 14 (Sondage 3) a livré un fragment de lèvre attribuable au XIII siècle, tout comme le mobilier issu du trou $\mathrm{F} 8$ du même sondage. Le trou F 12 (Sondage 6) a quant à lui fourni un élément céramique que l'on peut qualifier de "médiéval », tout comme le remplissage d'un des trous de poteaux du sondage 45 . 


\section{Une fosse remarquable}

\section{$\mathrm{xH}^{\mathrm{e}}$ siècles), constitué de pots sans anse à lèvre en bandeau, de pichets et de cruches} (fig. 8).

31 Le pichet, seconde grande catégorie de vases, apparaît au cours du $\mathrm{XII}^{\mathrm{e}}$ siècle. Trois céramiques de ce type ont été trouvées sur le site ; l'exemplaire le moins fragmenté est un pichet à col haut et lèvre en bandeau (vase 12). La base de son col et sa panse sont décorées de petites bandes peintes de couleur rouge-orangé. Un autre bord a lui aussi un col allongé et une lèvre déversée aplatie (vase 1) ; sa pâte, plutôt fine, est de couleur blanc-beige. L'anse rubanée (vase 3), à pâte beige légèrement grésée, est caractéristique 
des productions de pichets élancés de type "Saint-Jean-de-la-Motte », diffusés à partir du XIII ${ }^{\mathrm{e}}$ siècle.

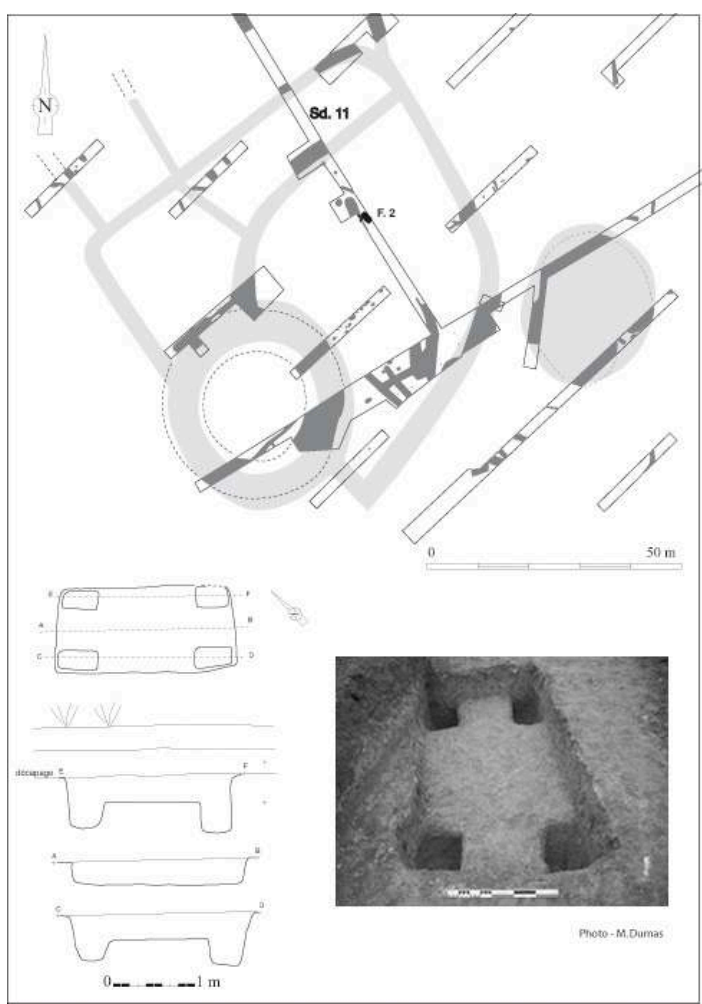

Figure 7 : Plan, profils et photographie de La fosse $\mathrm{F} 2$ (sond. 11).

Figure 7: Plan, sections and photograph of ditch F 2 (in trench 17).

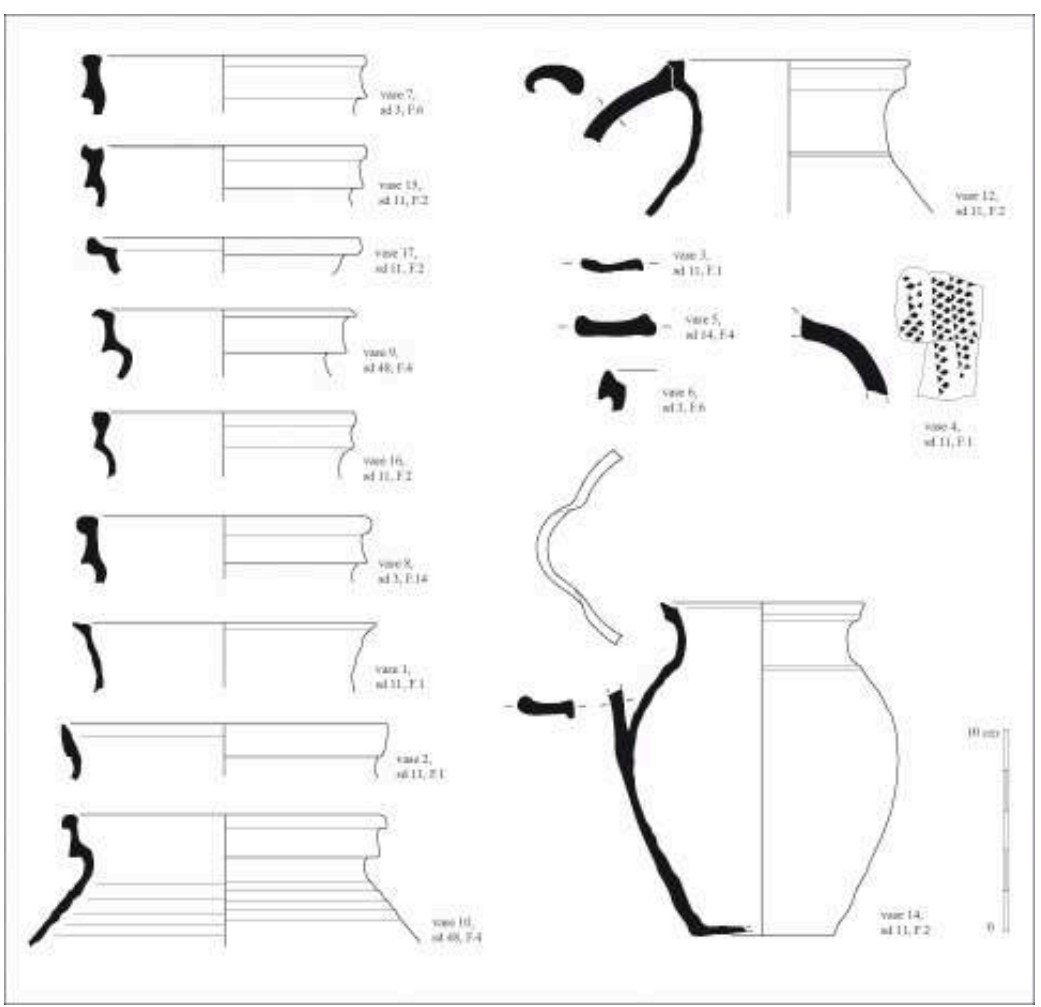

Figure 8 : Le mobilier céramique.

Figure 8: Ceramic assemblage. 

rares éléments protohistoriques ou de la période antique (fig. 10). Ils sont assez dispersés et restent, parmi les nombres d'indices d'occupation retrouvés, tout à fait anecdotiques. constitués d'abord d'un fossé de 7 à $8 \mathrm{~m}$ de largeur pour presque 2,5 $\mathrm{m}$ de profondeur. L'enclos qu'il limite et que l'on reconnaît parfaitement sur la photographie aérienne de G. Leroux enserre une zone de $20 \mathrm{~m}$ de diamètre. Cette zone principale est complétée au nord et au nord-est par un système de fossés dont les largeurs sont comprises entre 3 et $5 \mathrm{~m}$. Ils s'organisent, comme l'indique encore le cliché aérien, en une enceinte de presque $70 \mathrm{~m}$ de côté. 
40 Même si nous manquons de données stratigraphiques et d'éléments de datation, il semble qu'il y ait eu deux états pour cette basse-cour. La première, en forme de fuseau de $74 \mathrm{~m}$ de longueur pour une quarantaine de mètres de largeur, délimite une surface proche de $1600 \mathrm{~m}^{2}$. Ses fossés et celui de la motte (fig. 5) pouvaient être alimentés en eau par la mare située à l'est du site. En un second temps, la basse-cour aurait été augmentée dans cette direction par un nouveau fossé, parfaitement identifiable sur la photographie aérienne et que nous avons retrouvé dans les sondages 11 (F 5) et 56 (F 1). Après un angle droit au sud-ouest, cette nouvelle enceinte rejoint le fossé de la motte. Ce second état de basse-cour, presque rectangulaire et qui mesure de 50 à $70 \mathrm{~m}$ de côté, enserre ainsi près de $2500 \mathrm{~m}^{2}$, surface où apparaissent des trous de poteau et quelques fosses.

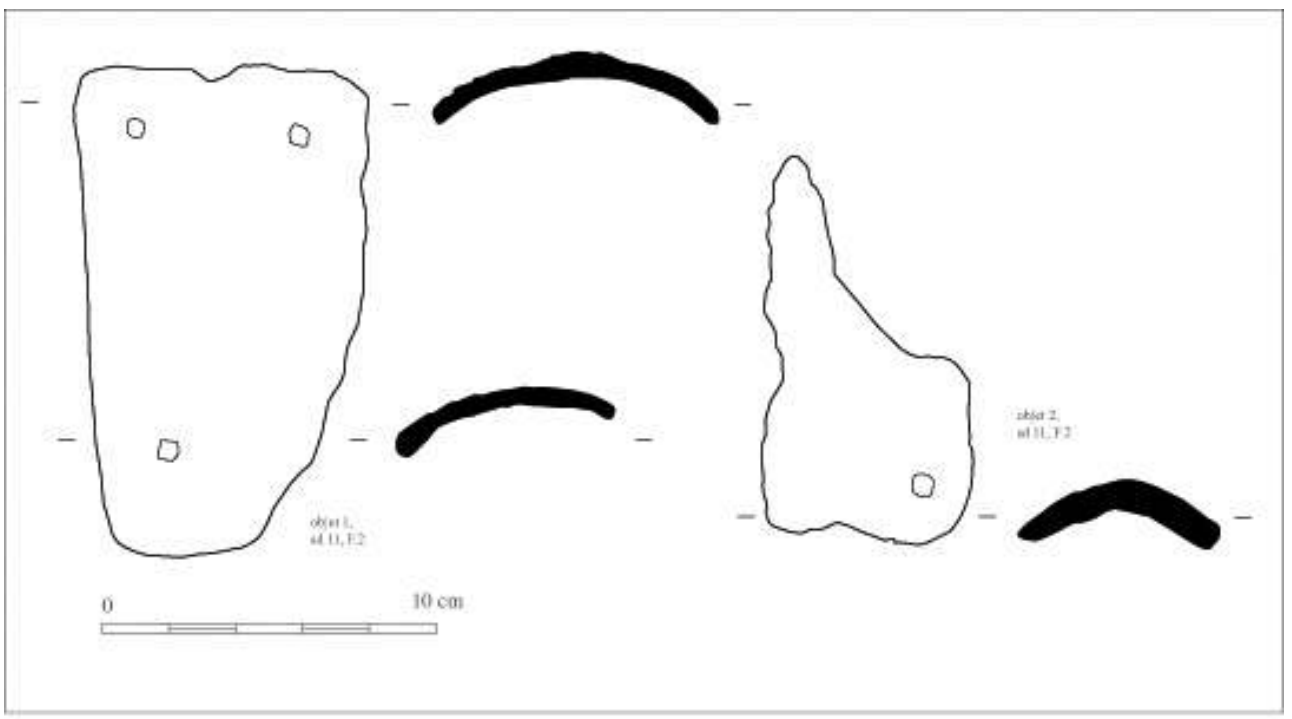

Figure 9: Les deux plaques de fer perforées, probables éléments de broigne ou d'armure. Figure 9: The two perforated iron scales, probable elements of armour. 


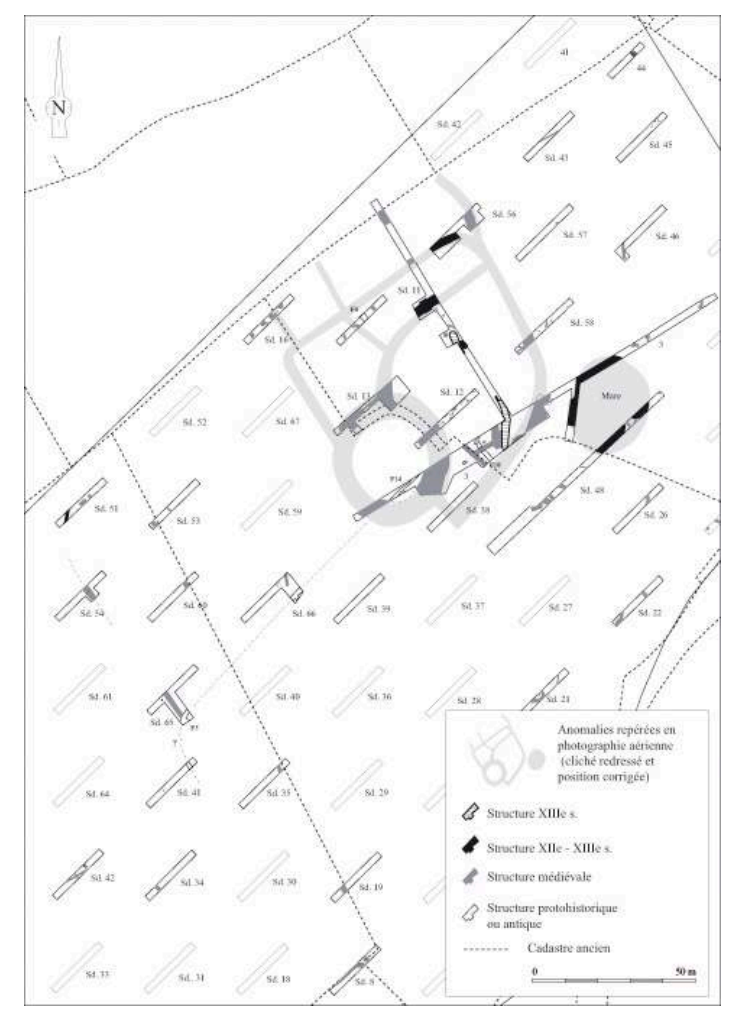

Figure 10 : Plan synthétique du site et phasage des structures testées au cours de l'opération. Figure 10: Overall plan and phasing of excavated features structures.

D'autres fossés de moindre dimension semblent en compartimenter l'intérieur. Quelques limites parcellaires complètent encore le plan vers le nord-ouest où elles pourraient organiser l'environnement immédiat de la motte et de sa basse-cour. En dehors des fossés, les structures sont essentiellement constituées de trous de poteaux. Les plus nombreux apparaissent à l'intérieur de l'enclos rectangulaire non loin de l'enclos principal.

Le mobilier céramique recueilli atteste d'une occupation centrée sur les $\mathrm{XII}^{\mathrm{e}}$ et $\mathrm{XIII}^{\mathrm{e}}$ siècles. Si aucun élément $\mathrm{du} \mathrm{XI}^{\mathrm{e}}$ siècle n'est à signaler, le site semble en revanche encore en activité pendant une partie du XIV ${ }^{e}$.

La caractérisation de l'occupation, au vu de ce mobilier, atteste d'abord d'activités domestiques. Les informations sont toutefois beaucoup trop lacunaires pour qualifier le statut des habitants de Guéramé, si ce n'est peut-être la présence d'un probable élément de broigne ou de plaque d'armure, type d'artefact que l'on trouve plutôt dans des contextes «privilégiés » de la fin du haut Moyen Âge et jusqu'aux XII ${ }^{\mathrm{e}}$-XIII ${ }^{\mathrm{e}}$ siècles.

\section{Ce que disent les textes (L. S.)}

Les résultats de ce diagnostic nous ont conduits à lancer une étude documentaire. Elle avait notamment pour but de mieux appréhender le contexte historique dans lequel avait pu évoluer cette motte de Guéramé et éventuellement d'identifier les lignages de chacun des complexes castraux recensés à Courgains et dans les environs. D'autres part, l'existence d'une ligne de fortifications liée à Robert II de Bellême, appelée «Fossés Robert» et qui passerait tout à côté du site de Guéramé nous a conduit à 
« revisiter » ce sujet qui, nous le verrons, relève sans doute plus de la légende que de faits avérés...

\section{La seigneurie de Guéramé}

On peut selon toute vraisemblance rattacher le site castral à une seigneurie médiévale dénommée Guéramé, toponyme qui perdure aujourd'hui par la désignation d'un hameau se trouvant non loin du site vers le nord-ouest. L'adéquation en question est manifeste grâce à l'étude des sources archivistiques. En premier lieu l'emprise du site castral se situe, au XVIII ${ }^{\mathrm{e}}$ siècle, sur des terres portant le nom de Guéramé (ADS, E 316-13). D'autre part un lignage de Guéramé est attesté depuis le xII ${ }^{e}$ siècle et jusqu'au $\mathrm{xv}^{\mathrm{e}}$, période à laquelle il fusionne avec celui de Concé. Ce dernier lignage est par ailleurs à mettre en relation avec la présence d'une ferme homonyme située à proximité immédiate du site (ADS, E 316-14). On peut du reste encore y voir aujourd'hui une autre motte castrale ceinte de fossés en eau. Enfin, les propriétés et les prébendes se rattachant aux différents membres de la famille sont localisées dans la région de Courgains.

\section{Le lignage de Guéramé}

Les archives, constituées en grande partie de sources diplomatiques (Charles et Menjot d'Elbenne 1886, p. 562-1184 ; Chédeville, 1968 ; Merlet, 1883), permettent de suivre le lignage de Guéramé sur cinq générations, depuis le début du XII siècle jusqu'à celui du $\mathrm{XIII}^{\mathrm{e}}$. Les sources écrites parlent indifféremment de Guéramé, Guerane, Guiraine ou Vado Ramato. Elles montrent une famille de la petite aristocratie du haut Maine dont les cadets sont destinés à la vie ecclésiastique. Parmi ces clercs se détache, au $\mathrm{XII}^{\mathrm{e}}$ siècle, la personnalité de Robert de Guéramé : par sa réussite sociale en premier lieu puisqu'il devient abbé de Saint-Vincent du Mans, mais également par les relations privilégiées qu'il entretient avec l'évêque du Mans, Guillaume de Passavant.

Aubert est le premier personnage du lignage à apparaître dans les textes, en 1128. C'est un laïc, probablement un miles. Il est dit "homme de Guéramé » (vero de Guéramé). Certainement âgé à cette date, il est sur le point de devenir moine (factus monachus). De sa femme Rohes, il a eu cinq fils : Guérin, Olivier, Robert, Payen et enfin Guillaume, déjà décédé à cette date.

48 Ainsi, parmi ces cinq fils, le personnage le mieux connu est Robert de Guéramé. Il est cité comme clerc en 1128 puis comme prieur claustral de l'abbaye Saint-Vincent du Mans en 1135. Il dirige par la suite ladite abbaye de 1148 à 1185 (Colomb, 1749). L'abbé Robert, ami et conseiller de l'évêque du Mans Guillaume de Passavant, passe pour avoir gouverné le monastère manceau avec beaucoup de sagesse et de prudence, tout en faisant preuve d'une grande fermeté dans la défense des intérêts de l'abbaye (ibid.).

49 L'abbé Robert a deux neveux : Robert et Guérin. Robert, bien que n'étant pas dans les ordres, obtient le bénéfice de la cure de Saosnes par son oncle, puis il est curé de René en 1191. Guérin, quant à lui, est un laïc dont le décès est attesté en 1208. 
50 Le fils de Guérin, Jean de Guéramé, est un clerc. Il cède des terres sises notamment à Courgains au profit de l'abbaye Saint-Vincent, par don en 1208 mais aussi par vente en 1210. En 1218, il est en pèlerinage en Espagne.

51 Le neveu du clerc Jean de Guéramé se prénomme également Guérin. Sa mère et lui sont en procès avec Jean au sujet de terres à Courgains, puis les protagonistes abandonnent leur différend en faisant don des biens en question à l'abbaye Saint-Vincent (1218). La même année, Guérin renonce à contester à l'abbaye la propriété d'autres terres à Courgains.

52 Les sources diplomatiques, d'origine ecclésiastique, permettent certes d'identifier le lignage de Guéramé, mais uniquement de manière indirecte puisque ce sont les cadets, destinés à la vie religieuse, qui apparaissent le plus souvent dans les textes. Le positionnement de la famille de Guéramé dans le réseau des solidarités vassaliques nous échappe presque totalement dans la mesure où, en premier lieu, il n'est pas perceptible par le biais des sources ecclésiastiques consultées alors que, d'autre part, nous n'avons pas retrouvé de titres féodaux concernant précisément le fief de Guéramé.

53 Tout au plus peut-on noter, toujours grâce aux sources ecclésiastiques, un lien de dépendance indirect envers la famille de Bellême. L'environnement dans lequel évoluent les membres de la famille de Guéramé au XII ${ }^{\mathrm{e}}$ siècle est en effet dominé par la présence de l'abbaye Saint-Vincent du Mans ou par des institutions en lien direct avec elle (prieuré de Courgains dépendant de ladite abbaye, cures de Saosnes et de René à la présentation des religieux de la même abbaye). Le sort de l'abbaye Saint-Vincent, fondée par l'évêque du Mans au $\mathrm{VI}^{\mathrm{e}}$ siècle et dont les possessions sont pour la plupart réparties dans le Saosnois, est étroitement lié au pouvoir épiscopal, que se transmettait la famille de Bellême d'oncle à neveu aux $\mathrm{x}^{\mathrm{e}}-\mathrm{XI}^{\mathrm{e}}$ siècles, quand ses membres n'étaient pas directement à la tête de l'abbaye à l'instar d'Avesgaud de Bellême au milieu du $\mathrm{XI}^{\mathrm{e}}$ siècle.

\section{La seigneurie de Concé et la fusion des lignages}

Les sources archivistiques font apparaître un lignage de Concé à partir du début du XIV ${ }^{\mathrm{e}}$ siècle : un acte daté de 1312 a pour objet la succession de la veuve de Phelipe de Concé (ADS, H 850). En 1401, Thomas Brissoul, écuyer, époux de Jeanne Pesaz était seigneur de Concé et de Sonnais. Au milieu du Xv siècle, les seigneuries de Guéramé et de Concé fusionnent : Jeanne de la Beillière est dite " dame de Concé » en 1452, 1455 et 1459 mais elle est dite également "dame de Concé et de Guéramé » en 1455 (Menjot d'Elbenne et Denis 1907 ; Chambois 1933 ; ADS, 4 J 383).

\section{Une ligne de fortifications médiévales aux confins du Maine et du Perche}

Le site de Guéramé se trouve à proximité immédiate d'une ligne de retranchement, longue de plusieurs kilomètres, dont une ancienne tradition populaire attribue la création au comte Robert II de Bellême à la fin du XI siècle (fig. 11). 


\section{Les « Fossés-Robert » et les forteresses reconnues}

56 $\mathrm{XVII}^{\mathrm{e}}$ siècle. Bry de la Clergerie (1620), en contant l'affrontement de 1098 entre Hélie, comte du Maine, et Robert II, seigneur de Bellême, cite le passage de l'Histoire ecclésiastique d'Orderic Vital (1075-1142 ?) où ce dernier énumère les châteaux élevés ou renforcés par le seigneur de Bellême. L'historien continue en attribuant au même personnage la construction d'un rempart de barrage connu par la tradition orale et locale : «[...] et fist les grands retranchements et fossez de plus de trois lieuës qui se voyent encores à présent entre Memers et Beaumont, et que les païsans appellent les fossés de Robert le Diable.»

Cependant, Orderic Vital ne parle pas expressément d'une ligne fossoyée de fortification, mais plutôt de plusieurs points d'appuis disposés en profondeur. Arrêtons-nous tout d'abord sur les événements de 1098. Pour s'opposer au visées expansionnistes des Normanno-Bellêmois, Hélie de la Fléche, comte du Maine, fait bâtir un château à Dangeul, créant ainsi un nouveau point d'appui à une dizaine de kilomètres au nord-est du verrou que constituent les places de Ballon et de Beaumont. Il s'approche ainsi à $6 \mathrm{~km}$ de Courgains, paroisse contrôlée par la famille de Bellême. Bientôt les troupes normanno-bellêmoises tentent de répondre à l'initiative mancelle en voulant surprendre la garnison de Dangeul. Mais le comte Hélie, prévenu de l'avancée ennemie, place ses gens aux endroits où le passage des troupes est délicat et empêche la progression adverse. Le parti normand abandonne le plan précédent et choisit alors de renforcer ses bases en territoire bellêmois afin de mener des actions militaires sur les terres contrôlées par le comte de Maine.

Voici le texte d'Orderic Vital relatif aux événements, suivi d'une traduction du $\mathrm{XIX}^{\mathrm{e}}$ siècle: "Porro, perstrepens rumor adventum regis praevenit, et principalis ordinatio provinciales, competentibus armaturis munitos, ascivit, ac ad transitus aquarum, sepimque, difficilesque aditus silvarum in hostes coaptavit. Tunc rex inimicis nihil nocere potuit, sed, rancore stomachatus, ferocior in illos exarsit, et Rodberto ingentem familiam bellatorum suis in municipiis adunare praecipit, et copiosos pecuniae sumptus erogavit, unde municipia ejus vallis et muris ac multiplicibus zetis undique clauderentur, et bellicosis larga stipendiariis donativa largirentur. Belesmensis itaque munio, ad haec promptus, oppida nova condidit, et antiqua, praecipitibus fossis cingens, admodum firmavit. Hic nimirum novem in illo comitatu habuit castra : id est Blevam et Peretum, Montem de Nube et Soonam, Sanctum Remigium de Planis et Orticosam, Allerias et mtam Galterii de clincampo, Mamerz, et alias domos firmas quamplurimus » (Vital, livre X, ch. VII).

Revue archéologique de l'Ouest, 27 | 2010 


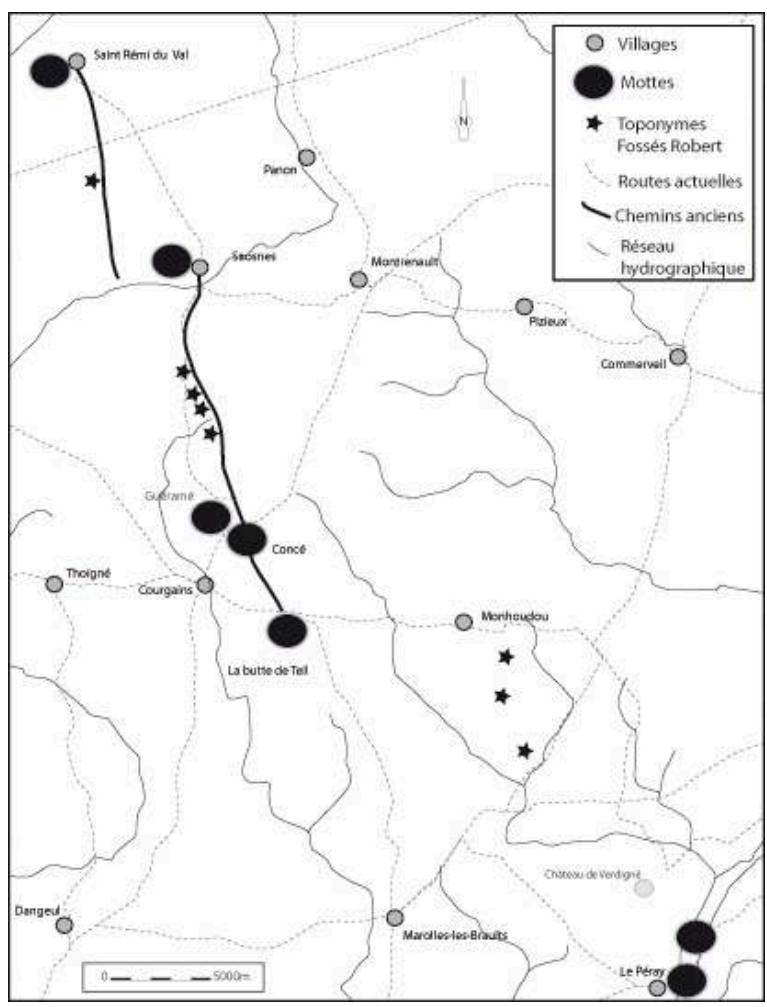

Figure 11 : Mottes et toponymes « Fossés Robert » dans la région de Courgains (d'après les données de J.-P. Darlot).

Figure 17: Mottes and "Fossés Robert" toponyms in Courgains area.

«Cependant la renommée prévint l'arrivée du Roi ; les ordres du comte du Maine firent lever en armes les troupes du pays, et on les plaça convenablement pour inquiéter l'ennemi aux passages des rivières et des fossés, ainsi que dans la traversée difficile des forêts. Le Roi donc ne put faire de mal à ses ennemis. Enflammé de dépit, il devint plus cruel contre eux; il ordonna à Robert de rassembler beaucoup de troupes dans ses places; il lui fournit de fortes sommes d'argent pour les fermer de palissades, de murailles et de toutes les constructions nécessaires, et pour qu'il payât largement les soldats qu'il emploierait. C'est pourquoi le comte de Bellême, ingénieur actif, bâtit de nouvelles places et fortifia beaucoup les anciennes, en les entourant de fossés escarpés. Il eut bientôt neuf forteresses dans son comté, savoir : Blèves, Peray, le Mont-de-la-Nue, Saosnes, Saint-Rémy-du-Plain, Ortieuse, Aillières, La-Motte-de-Gaultier-de-Clinchamp (commune de Chemilli), Mamers et plusieurs autres » (Vital, 1835).

Ces neuf places fortes citées par le chroniqueur sont disséminées dans le Maine (Saosnois) et le Perche, mais seules trois d'entre elles peuvent être rattachées aux Fossés-Robert: Saint-Rémi-du-Val, Saosnes et Peray (fig. 11). En revanche les prospections archéologiques menées depuis le XIX siècle ajoutent quatre autres sites d'importance variable: Concé, Le Mont-de-la-Garde, La Motte, et le château de Verdigné.

\section{Parcours et configuration des Fossés-Robert}

61 La première cartographie des Fossés-Robert a été réalisée par B. Jaillot en 1706. Du sud au nord, le tracé commence à Avesnes-en-Saosnois, rejoint Monhoudou, endroit où il s'interrompt jusqu'à Saint-Calais-en-Saosnois. De là il reprend son cours, passe entre le 
marécage de Gué-chaussé et le bourg de Saosnes pour suivre une courbe vers le nordest et aboutir à un étang au sud de Vezot. Ce tracé, qui passe nettement à l'est des sites de Guéramé et de Concé, a été jugé fantaisiste par les érudits du xix siècle qui ont proposé un parcours différent.

Ce nouveau tracé, initié par J.-R. Pesche puis repris par G. Fleury (1887), couvre $16 \mathrm{~km}$ et est ininterrompu depuis Peray au sud jusqu'à son terme à Saint-Remi-du-Plain au nord. Passant près de Guéramé et de Concé, il a fait l'objet d'observations de terrain et de confirmations toponymiques par les deux auteurs. S'il apparaît comme le plus assuré, ce dernier tracé n'en demeure pas moins problématique dans les secteurs où il a entièrement disparu, sans compter que d'autres sources cartographiques peuvent encore brouiller les résultats des investigations. Ainsi la carte de Cassini (1769, n 63) fait-elle figurer un lieu-dit les Fosses-Robert à l'ouest du tracé en question, plus précisément au nord-ouest de Toigné.

La première description précise des Fossés-Robert date du milieu du XIX ${ }^{e}$ siècle. À cette époque, les parties les mieux conservées de l'ouvrage sont situées entre Saosnes et Courgains : " Les portions encore entières, ou du moins apparentes, entre Saosnes et la butte du Teil, sont au nombre de 12 à 13, formant une étendue de $3 \mathrm{~km}$ environ, larges et profondes de 4 à 6 mètres. La hauteur du vallum ou talus, varie de 2 à $4 \mathrm{~m}$. On conçoit que, depuis 4 ou 5 siècles que ce retranchement a cessé d'être entretenu, le fossé a dû se combler, par l'effet des pluies et par l'entrainement des terres du talus; et qu'il est extraordinaire de lui trouver encore près d'un mètre de profondeur, et jusqu'à $6 \mathrm{~m}$ de largeur sur quelques points. » (Pesche, 1841 p. 804).

64 À la fin du XIX $x^{e}$ siècle, ces traces sont encore visibles au sud de Saosnes près des fermes de la Bélière et de Mont-Josson (alors que, pour le reste, les fossés ont quasiment disparu). G. Fleury (1887) a effectué deux relevés précis de ces sections de fossés qui ont été détruites en 1982 (Louise, 1990, p. 48).

G. Fleury décrit l'ouvrage de la manière suivante : le talus est à l'ouest du fossé, ce qui supposerait que l'ouvrage soit destiné à se protéger d'une intrusion venant de l'est. Toutefois un certain nombre d'éléments font douter de son caractère réellement défensif : le tracé se situe à mi-pente, le fossé est parfois surplombé par le côté ennemi, l'ouvrage semble être sans plan déterminé, ondulant sans motifs apparents et négligeant d'utiliser des points forts naturellement fournis par la topographie

\section{Datation et vocation de l'ouvrage}

La controverse au sujet de l'origine et de la vocation des Fossés-Robert remonte au $\mathrm{XIX}^{\mathrm{e}}$ siècle.

- Pour J.-R. Pesche (1841, p. 761), les fossés sont d'origine romaine et ont été par la suite réhabilités par Robert II de Bellême.

- G. Fleury (1887), pour sa part, pense que l'ouvrage doit bien sa réalisation à Robert II, grand spécialiste des ouvrages de fortification, et que les fossés forment une voie de communication reliant entre elles les forteresses du seigneur de Bellême à la manière des brachia romains évoquées dans la Guerre des Gaules (César, VII, 36) 3 .

-P. Cordonnier (1965) n'exclut pas de faire remonter les fossés à la protohistoire dans la mesure où ils relient des forteresses anciennes comme Peray et le Mont-de-la-Garde.

- G. Louise (1990) émet l'hypothèse d'une frontière matérialisant au sol la limite de la seigneurie de Bellême tout en détournant les assaillants vers des points de passage 
contrôlés. L'auteur remarque néanmoins que les possessions des Bellême s'avancent de trois à quatre kilomètres à l'ouest de cette ligne de démarcation et qu'en outre la ligne de fossés correspond davantage à la limite entre les donations faites à l'abbaye Saint-Vincent du Mans respectivement par les vicomtes du Maine et par les seigneurs de Bellême, limite fixée par accord dans les années 1060-1070 (Louise, 1990, p. 48).

\section{Les forteresses ponctuant les Fossés-Robert}

Du nord au sud les sites fortifiés suivants se rencontrent le long des Fossés-Robert :

-Saint-Rémi-du-Plain (actuel Saint-Rémy-du-Val): situé près de l'église paroissiale, ce château est presque la copie du château de Gisors construit à la même époque et par le même architecte, Robert II de Bellême. Il est composé d'une motte vaguement quadrangulaire surmontée d'un donjon de pierres de plan hexagonal avec contreforts aux angles. L'esplanade de la motte était fermée par une ceinture de murs flanqués de tours. Le dispositif est complété par deux enceintes successives comprenant fossés et talus. Ce château a joué un rôle militaire jusqu'au milieu du xve siècle (Fleury, 1887). L'ensemble, établi sur une pente inclinée vers le nord-ouest à partir du plateau portant le bourg, couvre quatre hectares.

- Saosnes : la forteresse se compose d'une motte surmontée d'un donjon en pierres sur plan barlong revêtu d'un appareil en opus piscitum. La motte est entourée d'un fossé avec talus, sauf vers le sud où la présence d'un marais constituait une défense naturelle. L'enceinte enveloppe le bourg actuel (Fleury, 1887). Il est à noter que Saosnes (Saugonna) est un vicus publicus au VII siècle (Garnier, 2004, p. 24).

- Le Mont de la Garde ou Butte du Teil : construit sur une colline dominant la plaine d'environ $100 \mathrm{~m}$, il est limité par une vaste enceinte de $250 \mathrm{~m}$ de diamètre, celle-ci étant composée de simples talus sur les côtés - suffisants vu la raideur de la pente - et d'un fossé avec talus à l'opposé de la motte située au nord-est de l'enceinte. L'ensemble donne au site une configuration d'éperon barré additionné d'une motte ultérieurement. Une situation naturellement très forte et le faible éloignement du château de Dangeul font que, même s'il n'est cité par aucune source, on peut raisonnablement penser que cet ouvrage a été utilisé par Robert II de Bellême dans sa lutte avec le comte du Maine.

- Concé : il s'agit d'un fief dont les seigneurs sont attestés dès le début du XIV siècle. Une motte accompagnée d'une basse-cour (la cour de la ferme actuelle) indique probablement une origine plus ancienne, ce que confirme un plan terrier du XVIII ${ }^{e}$ siècle (ADS, E 316-13), où la parcelle concernée est dénommée « jardin de la motte ».

- La Motte : il s'agit d'un indice toponymique (Verdier, 1978) qui n'est confirmé sur le terrain par aucun élément.

-Verdigné : c'est un manoir enserré dans une enceinte quadrangulaire de $150 \times 175 \mathrm{~m}$ environ, composée d'un talus et d'une douve. C'est un site de plaine qui pourrait remonter à l'Antiquité (Verdier 1978).

- Peray : la forteresse de Peray, en dehors du bourg, se compose d'une motte et d'une enceinte en demi-lune flanquée de quatre tertres aux angles, saillant en dedans de l'enceinte et ayant pu supporter des tours. Le château est cité parmi les places fortifiées par Robert de Bellême en 1098. Néanmoins le site a été occupé dès l'Antiquité comme l'attestent les découvertes de plus d'un millier de monnaies romaines tout au long du XIXe siècle (Cordonnier, 1965, p. 59). Le détenteur de ce château, mentionné avant 1077-1079, est à l'origine un vassal du lignage de Bellême et en fut dépossédé par Mabille de Bellême (Louise, 1990, p. 39). Une motte 
simple avec fossés, de moindre importance, se trouve également dans le bourg, près de l'église.

\section{Les châteaux de la seigneurie de Bellême}

Dans sa thèse, G. Louise (1990) a dressé une chrono-typologie des sites fortifiés médiévaux du Saosnois. Ce qui frappe de prime abord c'est la très grande concentration de forteresses dans cette région du haut Maine comparativement aux autres possessions du lignage des Bellême, que ce soit dans le Perche ou en Normandie.

9 G. Louise distingue trois types d'installation de fortifications :

-1er type : les sites attestés avant 1040 , représentant $25 \%$ du total. Ce sont probablement des sites stratégiques anciens réutilisés. Occupant des sites d'éperon dominants, ils contrôlent les principales voies de communication ou commandent de vastes territoires.

- 2e type : les sites attestés avant 1080, majoritaires avec $30 \%$ du total. Ils obéissent encore à des préoccupations stratégiques, mais pour des raisons plus locales liées à des politiques seigneuriales. Ils correspondent à une phase pendant laquelle la territorialisation et la privatisation des pouvoirs se précisent.

- 3e type : les sites attestés entre 1080 et 1106 représentent les $45 \%$ restants. Ils se rencontrent soit dans les vieux terroirs du Saosnois, soit dans des terroirs neufs de défrichement ou en marge des vieux terroirs, ou encore près des grandes forêts-frontières. Cette véritable explosion castrale est peut-être à mettre en rapport avec l'affaiblissement du pouvoir ducal en Normandie après la mort de Guillaume le Conquérant ainsi qu'avec les luttes entre Angevins, Manceaux et Normands pour la possession du comté du Maine.

\section{Synthèse des données et conclusions}

\section{La motte de Guéramé : l'une des seules de la région explorée sur la totalité de son emprise}

En dehors de la motte fouillée sur le site de l'aula de Doué-la-Fontaine dans le Maine-etLoire (De Bouard, 1973-1974), de l'exemple de Brûlon en Sarthe (Guillier, 1994), de " la Forêterie » à Saint-Jean de Lignière (Pirault, 2004) ou du " Péray » à Vivy (Thooris, 2001), toutes deux en Maine-et-Loire, et du récent diagnostic sur le «Champ de la Haute-Cour » à la Chapelle-Saint-Rémy dans la Sarthe (Guillier, 2009), les opérations d'envergure sur ce type de sites demeurent exceptionnelles malgré leur nombre très élevé dans la région. En dehors de la Basse-Normandie où les expériences de fouilles sont plus nombreuses (Chedeville, 1987), le site de Guéramé est l'un des premiers dans la région à avoir été diagnostiqué sur l'ensemble de son emprise (motte et basse-cour).

Le complexe est établi sur un versant. La motte occupe un des angles d'une basse-cour qui semble avoir d'abord été de plan fusiforme puis barlong, ces deux états occupant respectivement environ 1600 et $2500 \mathrm{~m}^{2}$. Ce type de motte aux dimensions modestes (20 $\mathrm{m}$ de diamètre) a l'avantage d'être facile et rapide à construire comme le montre la tapisserie de Bayeux par exemple, les corvées pouvant être affectées à ces travaux de terrassements (Mesqui, 1991). On ne connaît rien de l'accès à ce type de motte; il pouvait se situer autant à l'intérieur qu'à l'extérieur de la basse-cour (Collectif, 1981). Pourtant implantés sur un versant, les principaux fossés du site étaient très probablement en eau, recevant le trop-plein de la mare. Cette remarque est confirmée 
dans la région étudiée par J.-C. Meuret (les confins de la Bretagne, du Maine et de l'Anjou), où toutes les mottes établies sur les versants et les zones de plateau ( $38 \% \mathrm{du}$ total) étaient dotés de fossés pouvant être mis en eau (Meuret, 1993, p. 374).

L'étude des limites cadastrales aux alentours de Guéramé permet par ailleurs d'associer des tronçons de haies à un réseau parcellaire concentrique autour du site (fig. 12). Les limites de cet ensemble semblent fossilisées sur les planches du cadastre napoléonien au sud, au nord et surtout à l'est où elles s'appuient sur la voirie ancienne déjà évoquée. À l'ouest en revanche, on ne sait si le chemin (l'actuel CV 2) remplit le même rôle... Ce terroir, dont la motte de Guéramé serait le centre, occuperait ainsi une cinquantaine d'hectares.

\section{Les Fossés Robert : mythe ou réalité}

73 La plupart des auteurs ont repris sans réelle critique les premières mentions de ces " fossés Robert », lesquelles ne remontent qu'au début du XviI ${ }^{e}$ siècle. Rares sont ceux qui ont en effet mis en doute la fiabilité de ces sources, fondées sur quelques toponymes parfois épars et sur la tradition orale. Qu'en est-il de la réalité et surtout de l'efficacité d'une telle fortification de $16 \mathrm{~km}$ de longueur? Seul P. Darlot (1996) s'est interrogé sur l'existence d'un tel aménagement. L'hypothèse qu'il suggère, celle d'un réseau parcellaire et viaire d'origine antique qui aurait organisé la région semble plus intéressante. Les axes et les chemins qu'il a mis en évidence auraient ensuite perduré bien au-delà de la période médiévale. Des mottes ont été érigées sur certains de ces chemins pour en assurer le contrôle mais aussi, et c'est le cas de la motte de Guéramé, pour prendre pied dans des secteurs de confins mal maîtrisés et délaissés. Ainsi, on peut considérer que la plupart des «fossés Robert » ne correspondent finalement qu'à des tronçons de chemins creux plus ou moins remblayés. La tradition aurait peu à peu attribué ces dépressions, très souvent de même orientation, à un seul et même fossé de fortification, type de terrassement monumental qui ne manque pas autour des nombreuses mottes castrales conservées dans la région (Saosnes, le Péray ou encore Saint-Rémi-du-Val). Cette confusion pourrait constituer l'origine de ce qui a ensuite été cartographié et écrit au XVII ${ }^{\mathrm{e}}$ siècle.

74 Ne peut-on d'ailleurs la comparer avec celle qui a perduré jusqu'au début du $\mathrm{xx}^{\mathrm{e}}$ siècle dans le sud-ouest de la Mayenne où d'énormes zones excavées implantées sur un même axe et bordées de talus avaient été repérées dans la région de Craon? Un auteur, bientôt repris par d'autres, a écrit qu'il s'agissait d'ouvrages défensifs édifiés par les Bretons pour se prémunir des armées de Charles le Chauve (La Pommerais, 1908). Depuis, on sait, notamment à la suite d'une étude du BRGM, que ces cavités, appelées "Miaules ", constituent les vestiges de carrières ouvertes tout au long des filons de quartz aurifères qui traversent cette région de confins depuis la Guerche de Bretagne jusqu'à Château-Gontier (Meuret, 1993, p. 228).

75 Pour les exemples qui nous occupent ici, il est difficile de savoir si l'implantation de ces mottes correspond réellement à une frontière entre les zones d'influences bellêmoise et mancelle ou même à la limite des donations faites à l'abbaye de Saint-Vincent du Mans (Louise, 1990). Sachant que ces zones de marches constituaient autant de secteurs stratégiques, on peut imaginer que la frontière a pu fluctuer au gré des retournements d'alliances et des nombreux conflits qui ont dû émailler l'histoire de cette région disputée au tournant des $\mathrm{XI}^{\mathrm{e}}$ et $\mathrm{XII}^{\mathrm{e}}$ siècles. 


\section{Conclusions générales} dans la mémoire collective à un réseau de fortifications établi par Robert II, seigneur de Bellême à la fin du $\mathrm{xI}^{\mathrm{e}}$ siècle. Ce chemin, devenu stratégique, aurait été jalonné par des châteaux à mottes dont les plus anciens sont mentionnés à partir de 1040 tandis que les plus récents remonteraient à la fin du $\mathrm{XI}^{\mathrm{e}}$ siècle. Comme le suggère G. Louise (1990), c'est sans doute avec le déclin du pouvoir normand qui suit la mort de Guillaume le Conquérant que des seigneurs de rang plus ou moins élevé ont pu s'émanciper. C'est peut-être dans ce contexte que l'on peut placer l'origine du site de Guéramé, qui a probablement été confié à un chevalier de rang modeste vu la taille de sa motte et l'emprise limitée de sa basse-cour ainsi que du terroir associé.

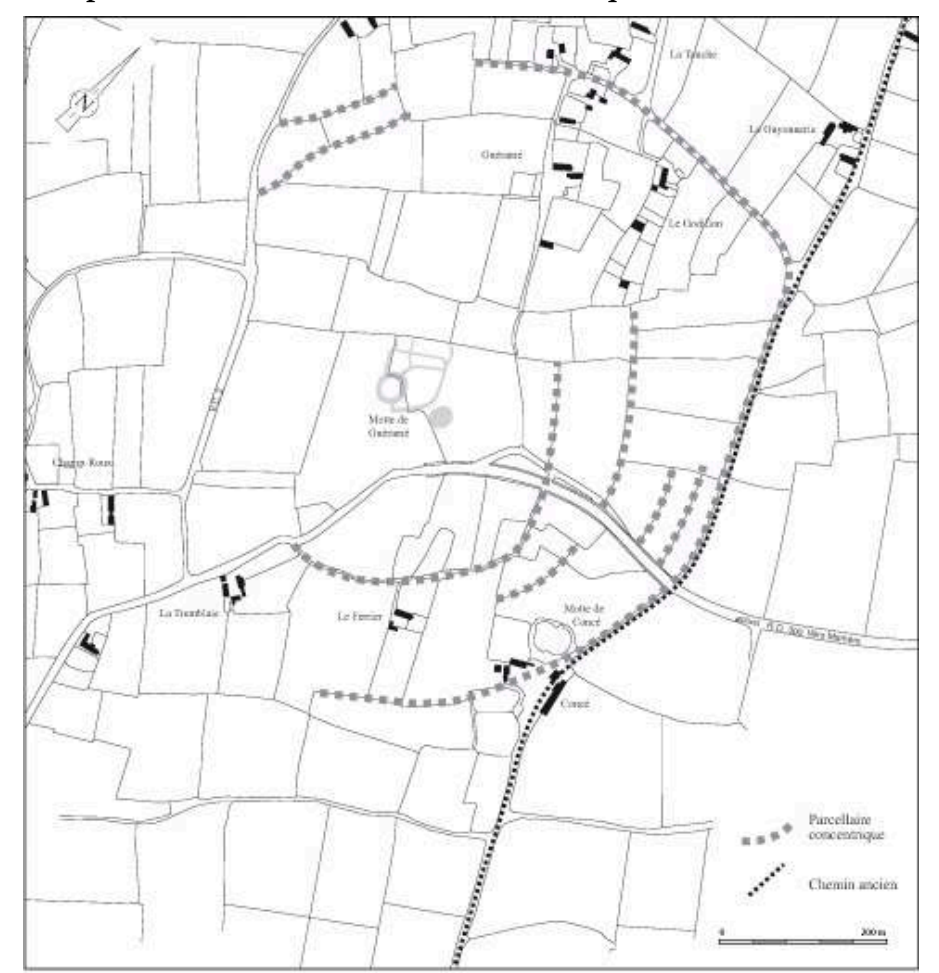

Fig. 12 - La motte de Géramé et son contexte parcellaire sur le fond cadastral de 1837 (feuille A3)

Figure 12: La motte de Guéramé et son environnement parcellaire sur le fond cadastral de 1837 (Courgains, feuille A3).

Figure 12: Guéramé motte and its field system on 1837 cadastral plan (Courgains, sheet A3).

Le mobilier céramique découvert atteste d'une occupation durant le XII ${ }^{\mathrm{e}}$ siècle, ce que les textes ne contredisent pas (apparition du premier membre du lignage de Guéramé 
en 1128). La création du site pourrait ainsi remonter à la toute fin $\mathrm{du} \mathrm{XI}^{\mathrm{e}}$ siècle ou aux toutes premières décennies du suivant.

Occupées jusqu'au XIII ${ }^{\mathrm{e}}$ siècle et pendant une partie du XIV ${ }^{\mathrm{e}}$, la motte et sa basse-cour ne semblent pas avoir perduré bien au-delà. Au milieu du $\mathrm{Xv}^{\mathrm{e}}$ siècle d'ailleurs, la seigneurie de Concé absorbe celle de Guéramé. Sur le terrain, alors que des vestiges de la première motte existent encore, rien ne subsiste en revanche de la seconde en dehors des quelques anomalies parcellaires encore visibles au XIX ${ }^{e}$ siècle. Il est probable que cette modeste motte de Guéramé ait été démilitarisée dès le $\mathrm{xv}^{\mathrm{e}}$ siècle, comme d'autres dans la région (Renoux, 2008), et qu'une grande partie de son emprise ait été remise en culture dès cette époque. Le site de Guéramé, malgré tout, a profondément marqué le paysage puisqu'au sein du terroir qui lui était directement associé, les orientations des fossés de la basse-cour rectangulaire se retrouvent très largement dans l'organisation générale du parcellaire, sous forme d'un réseau de haies qui a perduré jusqu'à la campagne de remembrement de la fin du $\mathrm{xx}^{\mathrm{e}}$ siècle...

\section{NOTES}

1. .BRGM, 1983 - Carte géologique de la France au 1/50 000, Fresnay-sur-Sarthe, $n^{\circ} 287$.

2. Sauf mention contraire, largeurs et profondeurs sont prises au niveau du décapage préliminaire (environ $0,8 \mathrm{~m}$ sous la surface actuelle du sol).

3. .César décrit l'un de ces «bras » lors du siège de Gergovie : « [...] il relia le petit camp au grand camp par un double fossé de douze pieds de large, afin que même des hommes isolés pussent aller de l'un à l'autre à l'abri des surprises de l'ennemi. » (traduction de L.-A. Constans, 1981, p. 281).

\section{INDEX}

Mots-clés : basse-cour, Motte castrale, plaques d'armure, tessons, trous de poteaux, fosse Keywords : armour scales, bailey, Castral motte, post-holes, post-reinforced pit, shards

\section{AUTEURS}

\section{ALAIN VALAIS}

Inrap - 20 rue Hippolyte-Foucault, 72200 Le Mans 


\section{LUDOVIC SCHMITT}

Inrap - 20 rue Hippolyte-Foucault, 72200 Le Mans

EMMANUELLE COFFINEAU

Inrap - 20 rue Hippolyte-Foucault, 72200 Le Mans

TRADUCTEUR_DESCRIPTION

MARC DUMA (TRADUCTION)

Inrap - 20 rue Hippolyte-Foucault, 72200 Le Mans

JEAN-FRANÇOIS NAULEAU (TRADUCTION)

Inrap - 4 rue du Tertre, 44477 Carquefou cedex 